Revue Française de Civilisation Britannique

\title{
La Reconstruction de la « civilisation britannique »: Bilan d'une pratique
}

British Civilisation Studies in a Time of Reconstruction: Some Observations

\section{Cornelius Crowley}

\section{CpenEdition}

Journals

Édition électronique

URL : http://journals.openedition.org/rfcb/2749

DOI : $10.4000 /$ rfcb. 2749

ISSN : 2429-4373

Éditeur

CRECIB - Centre de recherche et d'études en civilisation britannique

Référence électronique

Cornelius Crowley, "La Reconstruction de la « civilisation britannique »: Bilan d'une pratique », Revue Française de Civilisation Britannique [En ligne], XXIV-1 | 2019, mis en ligne le 22 mars 2019, consulté le 19 avril 2019. URL : http://journals.openedition.org/rfcb/2749; DOI : 10.4000/rfcb.2749

Ce document a été généré automatiquement le 19 avril 2019

\section{c)}

Revue française de civilisation britannique est mis à disposition selon les termes de la licence Creative Commons Attribution - Pas d'Utilisation Commerciale - Pas de Modification 4.0 International. 


\section{La Reconstruction de la « civilisation britannique »: Bilan d'une pratique}

British Civilisation Studies in a Time of Reconstruction: Some Observations

\section{Cornelius Crowley}

\section{Introduction}

1 Les notions de construction ${ }^{1}$ et de reconstruction invitent à un exercice de suivi longitudinal. Une reconstruction suppose la comparaison entre plusieurs états d'un même objet ou corps, le cas échéant pour constater un redressement. L'exercice est compatible avec la célébration du chemin parcouru et de l'œuvre accomplie. Il se prête aussi à des discours qu'on souhaite moins emphatiques ou complaisants. S'agissant de la civilisation britannique, les notions rendent possible l'interrogation d'un processus par lequel le champ s'est reconstruit et continue à se construire, dans le paysage universitaire des dernières décennies. Paysage, champ, aire : la terminologie renvoie à des opérations de démarcation: bornage, occupation, cadastrage. Elle présume une analogie entre le territoire académique, sa distribution des discours et des postures tenables, ses querelles, où on marche sur les plates-bandes d'une autre discipline, et le paysage...paysan. Rien là que l'application de la métaphore initiale par laquelle la culture se définit et s'interroge, les choses de l'esprit et les fruits des entreprises humaines prolongeant la rude culture des données naturelles: une affaire de sol, eau, semence, flore, faune, de rendements attendus, toujours aléatoires.

2 Il s'agit donc d'interroger la manière dont la civilisation britannique se construit. La forme pronominale évite certaines facilités. Elle indique que l'état actuel du champ ne se laisse pas expliquer par l'action décisive d'un acteur souverain hors champ ; pas, en tout cas, selon le schéma d'une causalité brutalement efficace. Et pas davantage par l'apport de quelques acteurs ayant réalisé une contribution majeure dans le champ. L'hypothèse adoptée ici, quant à l'agir politique et à la construction des savoirs dans les sciences 
sociales, est d'une conjoncture par définition moderne ${ }^{2}$, en aval donc du geste héroïque ou charismatique. Si la civilisation britannique se construit, c'est qu'elle ne se conçoit et ne se comprend, en tant que déploiement d'une intelligence et d'une action collectives -des personnes institutionnellement et socialement identifiées comme relevant du champ disciplinaire en question- ni comme un objet répondant au pilotage d'une institution (l'État: la tutelle) ni comme la simple réplique d'une conjoncture, celle d'une mondialisation à dominante anglophone.

Dans cet exercice de suivi longitudinal, nous passerons successivement par les notions d'agent et d'agentivité, puis d'environnement ${ }^{3}$ et de bordure institutionnelle, avant de nous pencher sur la structuration interne de la discipline comme aire ou comme champ d'étude, où se consolide un dessein d'autonomie fondé sur l'articulation heuristique entre un objet de recherche et une méthode. Nous postulons que cette articulation entre un environnement mis à distance, mais sur lequel on prend appui, et une plus grande affirmation d'agentivité intra-champ, se traduisant par l'approche méthodique de son objet, s'accompagne nécessairement d'une illusio porteuse, au sens où Bourdieu emploie ce terme ${ }^{4}$.

\section{Agent, agentivité}

Dans un passage de ses Méditations pascaliennes (1997), Bourdieu dessine les contours « des sociétés stables » :

Le charme indiscutable des sociétés stables et peu différenciées, lieux par excellence, selon Hegel, qui en a eu une intuition très aiguë, de la liberté concrète comme « être-chez soi » (bei sich sein) dans ce qui est, trouve son principe dans la coïncidence quasi parfaite entre les habitus et l'habitat, entre les schèmes de la vision mythique du monde et la structure de l'espace domestique par exemple, organisé selon les mêmes oppositions, ou encore entre les espérances et les chances objectives de les réaliser. Dans les sociétés différenciées elles-mêmes, toute une série de mécanismes sociaux tendent à assurer l'ajustement des dispositions aux positions, offrant par là à ceux qui en bénéficient une expérience enchantée (ou mystifiée) du monde social ${ }^{5}$.

5 Le " charme indiscutable des sociétés stables » opère par l'aptitude à induire une familiarité. Les occupants d'un tel espace peuvent se sentir chez eux et s'y mouvoir librement. Le tableau dessiné est celui d'un espace structuré comme un espace mythique, aussi simple que stable, pas encore reconstruit en un espace soumis à la régularité des procédures par lesquelles l'agent rendra des comptes à des instances d'habilitation et d'évaluation ${ }^{6}$. Dans un tel espace, le pouvoir exercé est d'autant mieux assuré qu'il s'impose comme une évidence indiscutable, fondée sur l'autorité des agents illustres. Un tel espace laisse du jeu, laisse du champ. Tel n'est pas le tableau du champ de la civilisation britannique aujourd'hui. Je ne dirais pas que j'ai connu pareille "société stable», en aval d'une nomination à un poste fléché civilisation britannique en 1992. Le moi, invoqué comme source de témoignage et comme gage incertain d'une sincérité présumée, est de peu d'utilité dans la construction d'une analyse portant sur un champ quelconque. S'agissant d'un «habitat » académique, où l'enjeu est celui de l'objectivation ${ }^{7}$ (comme fait social susceptible d'analyse) d'un champ d'exercice habité par des agents habilités à l'objectivation d'autres champs de l'espace social, le déploiement d'une réflexivité critique est au cœur d'un précédent livre de Bourdieu, homo academicus (1984) ${ }^{8}$. L'objectivation de son propre parcours personnel, à titre de contribution à un bilan 
longitudinal plus général, est éventuellement possible, mais à la condition de mettre entre parenthèses les notions de sujet ou de subjectivité, de passer par le relevé des conditions d'exercice et de service dessinant le schéma d'agentivité qui fut celui des études civilisationnistes vers la fin de la présidence de François Mitterrand, à l'orée de la décennie des années 1990. La deuxième condition est de doubler tout travail d'objectivation de soi par une objectivation externe, réalisée par d'autres. Cette règle de l'objectivation d'une pratique depuis une position hors champ, sans connivence possible, s'entend comme une nécessité implacable.

6 Une analyse longitudinale permet donc la reconstruction d'un processus que son propre parcours a permis de constater et d'observer individuellement. Ainsi la péremption des « sociétés stables et peu différenciées» aura-t-elle conditionné la promotion du champ civilisationniste. Cette péremption, pas encore achevée à la fin des années 1980, vingtcinq années après mai 1968, est désormais incontestable, depuis le début de ce nouveau siècle. Or, dans ce qu'il restait vers 1992 d'une telle " société stable ", l'agentivité dévolue à la civilisation britannique au sein d'un espace savant où il y avait encore un peu de jeu (comme dans le football pratiqué par l'équipe du Brésil en 1970 : pour quelque temps encore le terrain autorisait un jeu de seigneurs ${ }^{9}$ ), tenait du rôle de supplément : au service de l'étude de la langue et de la littérature, un éclairage utile. La fonction d'accompagnement reconnue à la civilisation britannique (ou américaine) ${ }^{10}$ supposait, dans l'ajustement entre "les habitus et l'habitat ", un degré de déférence méthodologique: l'hypothèse, prédominante à l'époque, d'une autonomie des œuvres dotées d'un statut d'exceptionnalité esthétique, qui configurait le versant littérature des études anglicistes, avait pour corrélat le positionnement subalterne et, au sens étymologique, vulgaire des savoirs contextuels, externes. S'agissant de l'expérience de la «liberté concrète comme "être-chez-soi »"11, dont l'œuvre singulière était le paradigme, si à l'occasion elle pouvait autoriser une exploration du territoire contextuel autour, cela faisait sens tant qu'elle restait animée de la liberté qui se déployait dans les œuvres. Moins autorisée, en retour, la lecture externe et dé-singularisante des œuvres, par extension et affirmation méthodologique du domaine civilisationniste ${ }^{12}$.

7 Le tableau que je dresse ici est celui d'un schéma directeur de la civilisation britannique, telle que je l'ai connue et pratiquée, en essayant de m'y faire une place. On peut trouver dans ce tableau des effets de simplification et de raccourci : la chose vue est en tous les cas tributaire des limites d'un point de vue situé. Cela est fatalement la limite, s'agissant de la reconstruction d'une configuration institutionnelle dans laquelle l'agent de l'université publique, ayant prétendu à un habitus dans un « habitat » académique, s'est inscrit. Je préciserai aussi les conditions localement contingentes d'un recrutement : par mutation et à la fin d'une journée pendant laquelle une commission de spécialistes avait examiné les candidatures à six ou sept postes (la mienne démontrant une adéquation incomplète au poste à pourvoir: une thèse irlandaise en littérature et trois années d'expérience en IEP). Une "objectivation $»^{13}$ de son propre profil ou habitus, de sa conformité à "l'habitat» de l'époque, atteste une adéquation fragile à l'intitulé disciplinaire proposé - civilisation britannique- ; fragilité jugée tolérable, en 1992, du fait de l'extension du nombre de postes à pouvoir (moment propice: la période socialiste, 1988-1993, du second septennat de François Mitterrand, celle de la mise en œuvre du projet Universités $2000^{14}$, qui a conduit à l'assouplissement transitoire des droits d'entrée). Assouplissement plutôt asymétrique, tolérable en $1992 \mathrm{du}$ fait de la position relative de la civilisation britannique au sein du champ angliciste, $11^{\mathrm{e}}$ section $\mathrm{CNU}$ : celle d'un champ 
encore subalterne, en un moment d'extension institutionnelle (quantitative) de l'anglistique dans les universités. Or c'est en constatant le redressement de la civilisation britannique depuis cette position ancillaire, encore manifeste en 1992, que l'on prend la mesure de la reconstruction d'une discipline sur une période courte, mais selon une logique d'une parfaite lisibilité, désormais achevée.

Ainsi un flottement entre habitus et le champ disciplinaire du poste (civilisation britannique) se laissait-il tolérer au sein d'un « habitat » à l'époque sous-réglementé et en expansion, qui admettait

une plus grande latitude quant au profil affiché et à pourvoir: le terme enseignantchercheur était à l'époque moins utilisé, l'autonomie des équipes d'accueil peu ou pas mise en avant par rapport aux UFR. L'objectivation des conditions d'une nomination, puis des conditions d'exercice dans le parcours qui a suivi, m'oblige donc à reconnaître avoir fait carrière depuis une conformité précaire entre habitus et habitat institutionnel, alors même qu'un processus de reconfiguration générale du paysage universitaire était en train d'induire des conditions d'exercice enfin propices au redressement de la civilisation britannique. Propices donc à la péremption des conditions de sous-régulation ayant rendu possible mon élection en fin de journée. En effet, on pouvait encore, en 1992, prétendre enseigner la civilisation irlandaise avec pour principale qualité déontologique son lieu de naissance et son statut d'Irlandais de service, de langue maternelle anglaise, aisément opérationnel dans les filières alors en forte expansion: LEA, bi-filières droitlangues, économie-langues.

\section{Environnement}

10 La démarche adoptée ici passe par une approche externe : faire l'inventaire d'une série de données qui, de prime abord ou en leur intention, n'ont rien à voir avec le contenu des savoirs académiques, mais ont assurément à voir avec les conditions de leur production. En effet, l'hypothèse de l'autonomie souveraine des savoirs est concomitant du maintien, le plus longtemps possible, d'un voile d'ignorance quant au conditionnement (qui n'est pas la détermination servile) des savoirs par leur environnement institutionnel. Et quand bien même le voile est levé, c'est généralement pour la présentation des mutations institutionnelles comme autant de mesures à déplorer ${ }^{15}$. En préalable à l'analyse de la civilisation britannique comme champ disciplinaire, nécessairement doté de méthode(s) car doté d'objet(s), nous examinons les infléchissements de l'environnement institutionnel, en relation avec lesquels le champ disciplinaire a dû se consolider. Notre hypothèse est que l'environnement institutionnel externe a été propice à la consolidation de la civilisation britannique comme champ de recherche et d'enseignement spécifique ; que les modifications (réformes) dont nous faisons l'inventaire ont pu infléchir les conditions d'exercice et de consolidation de la discipline. Ces différentes modifications auront par ailleurs contribué à l'extinction de la configuration antérieure, dont la stabilité est corrélée au « charme ». Il est cohérent que les mesures institutionnelles aient suscité des discours faits de déploration d'une perte. Cependant, s'agissant des conditions de reconstruction de la civilisation britannique, il me semble important de reconnaître que les mutations intervenues dans l'environnement institutionnel et dans le champ disciplinaire appellent une lecture qui s'affranchit des discours charpentés par une intentionnalité trop simple, où les effets de normalisation ou de rationalisation, indiscutables, seraient univoques dans leurs desseins, qui seraient forcément négatifs. 
Citons donc les modifications suivantes, dont l'effet d'ensemble est d'établir une configuration profondément changée.

1) La Réforme Bayrou (1997), avec introduction d'une semestrialisation de l'année universitaire ${ }^{16}$. Aucune relation directe avec les contenus enseignés, avec la répartition des différents champs au sein d'une même discipline, ici celle des études anglaises. Cependant l'organisation des enseignements sur un seul semestre tend à la spécialisation et clarification des éléments appelés à figurer dans l'architecture des formations proposées. Le paysage tendra donc, à partir de 1997, vers une définition plus précise des champs et des savoirs. Or dans un paysage jusqu'alors sous-défini, où le rôle dévolu à la civilisation britannique pouvait être ancillaire, cette plus grande spécialisation sera propice à son émancipation. De nouveau, citons Bourdieu dans les Méditations pascaliennes :

Il est certain que la connaissances des grandes tendances de l'évolution scientifique élévation progressive des droits d'entrée, accroissement de l'homogénéité entre les concurrents, diminution de l'écart entre les stratégies de conservation et les stratégies de subversion, remplacement des grandes révolutions périodiques par les multiples petites révolutions permanentées, libérées des causes et des effets politiques externes, etc. implique et induit une définition normative de la loi fondamentale d'un champ réellement scientifique, à savoir le consensus sur les objets légitimes du dissensus et sur les moyens légitimes de le régler ${ }^{17}$.

13 2) La semestrialisation est au départ une simple affaire de calendrier: deux semestres comme structuration calendaire, au lieu d'une année universitaire, un alignement sur les normes internationales. Elle collabore donc (indirectement) à une telle «évolution scientifique ${ }^{18}$, dont la manifestation intra-disciplinaire se traduit en effet par une « élévation progressive des droits d'entrée ». L'élévation est le concomitant d'une consolidation disciplinaire. Tout cela est-il à mettre en relation avec la semestrialisation? Oui, modestement, imperceptiblement. Les infléchissements systémiques sont des infléchissements en apparence mineure. Cette première réforme dans l'architecture des formations est en phase avec d'autres mutations dans l'environnement institutionnel. La configuration fait sens. Mais cela ne veut pas dire que sa compréhension relève d'une lecture intentionnaliste ${ }^{19}$.

14 3) Les accords de Bologne, avec adoption d'une unité de compte permettant l'expression des enseignements (de l'offre de formation) sous la forme de leur valeur ECTS, l'ensemble se traduisant par la mise en place d'un espace européen des formations, fondé sur la portabilité des crédits ${ }^{20}$.

15 4) La mise en place par le Ministère de l'enseignement supérieur du schéma LMD $^{21}$ en application de ces accords, accompagnée d'une rationalisation des procédures d'habilitation des diplômes : rôle dévolu à l'AERES ${ }^{22}$ puis au HCERES ${ }^{23}$ dans l'évaluation, conduisant à la présentation et la justification d'une offre de formation à travers son adossement à la recherche, la procédure conduisant à une plus stricte conformité à des normes académiques nationales et internationales dans le libellé des cours, notamment pour cause de l'impératif de lisibilité, à la fois pour les évaluateurs et pour les différents acteurs et usagers de l'espace européen. Ainsi les configurations locales, relevant d'un état précédent du paysage universitaire, ont-elles tendance à dépérir dans ce nouveau cadre. 
16 5) L'évaluation et l'habilitation des laboratoires/équipes d'accueil selon des procédures conduisant à une plus grande formalisation des axes de recherche.

17 6) La visibilité progressivement prise par l'IUF (créée en 1991) ${ }^{24}$ dans le paysage universitaire; la plus grande sensibilisation aux procédures de délégation CNRS à la disposition des universitaires, sur projet de recherche déposé.

18 7) La mise en place d'un paysage de recherche fondé sur l'appel à projets : Labex, Idex (2010-2011) ; ANR (2005) ${ }^{25}$, European Research Council (2007) ${ }^{26}$.

8) La Loi LRU (2007), qui tend à mettre en exergue, en prolongement des modifications intervenues depuis 1997, la double mission d'enseignant-chercheur, dont le profil, lors du recrutement puis en cours de carrière, suppose la conformité à un champ clairement identifié, condition de cohérence et de dynamisme (comptage comme publiant) en cours de carrière.

20 9) La mise en place, à partir de 2008, des comités de sélection, prenant la relève des commissions de spécialistes; comités désormais établis avec une composition ad hoc, déterminée par le profil du poste publié, et une parité entre membres internes à l'établissement recruteur et membres externes.

21 Ce qui a été dit plus haut ici à propos de la première de ces mesures, à savoir qu'il n'existe aucune relation directe avec les contenus enseignés, avec la répartition des disciplines ou des différents champs au sein d'une même discipline, ici le champ général des études anglaises, vaut pour les autres mutations mentionnées ici. Cependant l'ensemble établit une configuration qui conditionne l'activité en recherche et en enseignement, dans les différentes disciplines $d u$ paysage universitaire. Ces mesures ont modifié l'environnement institutionnel où s'inscrit l'anglistique, l'ensemble des paramètres régissant la production du champ de recherche de la civilisation britannique: les relations intra-champ, avec les autres éléments qui ensemble sont constitutifs de la $11^{\mathrm{e}}$ section CNU ; les relations inter-disciplinaires, avec des champs connexes, l'histoire, la sociologie, ou les sciences politiques. Sans oublier les relations avec d'autres institutions universitaires au sein d'un paysage universitaire européen, dont fait indiscutablement partie l'université britannique, dans une conjoncture que nous sommes fondés à débuter avec la mise en place progressive de l'Acte unique (1986), puis le Traité de Maastricht (1992). Et sans oublier les réseaux internationaux où s'inscrivent désormais projets de recherche et possibilités de publication, la civilisation britannique étant placée dans une situation particulière: en relation privilégiée avec les universités britanniques, stratégiquement incontournables dans le montage des projets européens; tout aussi incontournables pour les échanges intellectuels qui conditionnent le rayonnement de la recherche, dans le cadre des procédures d'évaluation. En position d'intermédiation donc, entre un monde anglophone et un bloc universitaire continental européen ${ }^{27}$. A cela, s'ajoutent des élargissements supplémentaires, s'agissant des domaines et des aires: élargissement de la recherche vers le global south, comme objet mais surtout comme foyer de projection des interrogations en retour, en un processus d'ouverture et de confrontation déployé depuis (et vers) les institutions de recherche du monde anglophone.

22 Rien d'inattendu dans cette liste de mutations institutionnelles. Elles font l'objet d'un vaste corpus de discours émis par le monde universitaire, généralement sous forme de critiques de la rationalisation gestionnaire ainsi mise en œuvre. Ces critiques sont cohérentes avec la déploration d'une perte de «liberté concrète $»^{28}$, d'une normalisation du 
champ et d'un assujettissement des chercheurs. Mon propos consiste simplement à émettre l'hypothèse que le rayonnement relatif, plus affirmé, de la civilisation britannique au sein du champ des études anglaises a pu être consolidé, en corrélation avec l'ensemble des mutations ayant reconfiguré l'environnement universitaire où s'exerce le métier d'enseignant-chercheur. Tel n'est pas forcément le cas, au cours de la même période, pour le champ d'ensemble, celui des études anglaises. Nous savons bien (leçon élémentaire de la méthode humienne) que corrélation n'est pas causalité. L'évitement des schémas d'intentionnalité ou de causalité linéaires permet une appréhension un peu plus juste du destin longitudinal du champ de la civilisation britannique. Quant à savoir si l'acquis est stable ou au contraire passager, c'est une autre question. Par prudence et par simple observation des mutations en cours, je dirais que rien n'est désormais solidement acquis.

\section{Aire/champ}

Area studies: domaine, aire, champ. Au risque d'être redondant, nous définissons le lieu d'exercice d'un projet de savoir par rapport à son extension et son terrain d'application. La discipline que nous interrogeons ici nomme l'étendue d'un champ de savoir, soumis à la règle méthodologique: un savoir dont l'assurance requiert de se donner des frontières et des limites, permettant ensuite un éventuel échange interdisciplinaire. Mais si la civilisation britannique, ou encore British studies ou anglophone studies, nomment le terrain d'étude, l'intitulé laisse en suspens l'indication quant à la méthode. Or l'« élévation progressive des droits d'entrée » passe par la précision des méthodes censées valoir pour une série d'objets qui sont présumés comparables, dont l'étude sera ainsi normalisée. Ainsi les méthodes s'appliquant à la civilisation britannique ne seraient-elles ni plus ni moins celles qui s'appliquent à d'autres aires : lusophone, francophone, arabophone. Mais est-ce si simple? La généralité d'un tel propos semble indiscutable et prudente: invocation d'une neutralité axiologique, postulat d'une généralité tout-terrain de la méthode. Mais cela suffit-il, dès lors qu'il s'agit d'étudier des aires de langue et de culture, dont la traductibilité interculturelle, pour le dire pudiquement, est diaboliquement complexe ${ }^{29}$ ? Et le postulat de la généralité et de la neutralité de méthode a souvent été oublié en route : " more honoured in the breach than in the observance $»^{30}$. Fallait-il être celte pour saisir l'âme celte, ou pour la saisir autrement que comme chimère, un artefact dérivé des essentialisations romantiques? Ou, pour formuler les choses de manière plus incisive, $y$ aurait-il une âme celte à saisir? Car le choix s'est souvent joué de manière binaire, entre l'hypostase d'une âme, insaisissable par ceux qui n'en vivent et n'en vibrent pas, et la réification de l'objet civilisationnel, étudié de l'extérieur, donc forcément à travers, sans compréhension autre que celle de la réification essentialisante. Les deux impasses sont solidaires et s'entretiennent. Et nous ne sommes toujours pas sortis des difficultés ayant présidé à la naissance des études par aires culturelles, études souvent rivales, sublimées par la différence des langues ${ }^{31}$.

Plusieurs droits d'entrée sont donc à acquitter, le moindre n'étant pas en vue de l'acquisition d'une réflexivité théorique requise lors de chaque exploration d'un terrain. Les procédures de construction d'un savoir, ainsi que la négociation des lieux de passage où les droits d'entrée sont exigibles, sont à la fois plus ouvertes et plus retorses que celles, institutionnellement plus normées, que mentionne Bourdieu. Une discipline s'appliquant à une aire (le champ de la civilisation britannique) devra ainsi acquitter plusieurs tickets 
d'entrée : histoire (sociale, politique, culturelle, des idées), sciences politique, sociologie ${ }^{32}$ - Au risque de devoir prétendre à une habilitation pluridisciplinaire, alors que la spécialisation pointée par Bourdieu tend vers l'établissement d'une compétence dans l'exploration de champs de plus en plus soumis à une culture disciplinaire intensive et pointue, garant d'une exclusion des entrants maverick. Ainsi la reconstruction de la civilisation britannique s'est-elle traduite par l'acquisition, de la part des nouvelles générations de chercheurs, de méthodologies mieux affirmées, davantage réfléchies.

Mais un acquis est toujours relatif et révisable. Il s'entend comme un fait contingent répondant aux possibilités d'un environnement spécifique, qui ne cesse d'évoluer et de produire d'autres variations. Ainsi dès lors que la recherche en civilisation britannique se revendique plus nettement de la méthode historiographique, elle s'ouvre à l'échange avec le champ général de l'histoire, à la transférabilité vers l'anglistique de "droits d'entrée » établis au sein de la discipline de l'histoire, sous condition d'avoir exploré un terrain se rattachant à l'aire des études anglaises. Même chose pour la sociologie générale ou pour la sociologie des religions.

Et ce n'est pas tout, si nous faisons l'inventaire des «droits d'entrée » qui sont exigibles, s'agissant de l'articulation entre un objet d'étude et sa méthode. Car dès lors que la discipline se définit comme aire, et qu'on récuse l'hypothèse narcissique d'une singularité inapprochable par ceux qui n'en seraient pas identitairement les émanations ou les surgeons, nous devons reconnaître que la logique mise en évidence ici, celle d'une reconstruction de la civilisation britannique comme terrain d'une étude méthodique, est traversée de dynamiques supplémentaires. En effet, alors que se poursuit la consolidation d'un champ disciplinaire affirmé, la dynamique des savoirs, à l'image de la dynamique des sociétés et des faits sociaux, laisse supposer qu'aucune stabilisation n'est désormais durablement acquise.

Prenons deux exemples d'articulation entre objet et méthode qui sont bien loin du cas, en apparence simple, de la civilisation britannique, dont la définition comme aire ne comporte aucune restriction à une seule ou unique méthode exigible. De la théologie, qu'il examine en sa relation à la philosophie, Denis Guénoun dit qu'elle est «l'étude de l'idée de dieu (ou de Dieu) $»^{33}$. La méthode de la théologie est donc difficilement séparable d'un postulat ontologique quant à son sujet/objet d'étude: le postulat de Dieu comme être. Notre domaine de la civilisation britannique (plus modeste, plus aisément attesté, territorialement et atmosphériquement) n'est pas suspendu à une telle pétition de principe : le monde anglophone, historiquement déployé, existe incontestablement, en amont de l'interrogation savante qui le reconstruit en l'explorant. Mais si on se réoriente, passant d'une discipline de terrain ou d'aire pour gagner, dans la configuration des sciences sociales, le champ spécifique des gender studies, alors on pointera une dynamique de requalification épistémologique, désormais en cours, sans doute susceptible d'aucun ralentissement ou stabilisation. En introduction à sa contribution au livre de Yan Brailowksy et Hervé Inglebert sur les sciences humaines ${ }^{34}$, Michèle Riot-Sarcey définit ainsi son objet d'étude, qui s'entend comme une méthode permettant une construction autre, renouvelée et critique, d'un champ institutionnellement normé, ici le champ disciplinaire de l'histoire:

Dans ces quelques pages, je souhaiterais non seulement éclairer le terme, mais surtout en présenter la pertinence épistémologique en fonction de l'exigence de son usage. Le domaine de l'histoire sera mon terrain d'investigation ${ }^{35}$. 
Si nous ne sortons pas de la série des métaphores prévisibles (terrain, domaine, solidaires de champ, frontière, aire), gender est emblématique d'une requalification épistémologique des terrains de recherche. Ainsi tout champ est-il susceptible d'une requalification, par adoption d'une perspective qui va éclairer et lire autrement la configuration d'un terrain déjà donné. Or la requalification d'un objet, par un effet de mutation épistémologique, atteste des reconstructions de fond qui sont en cours dans la disposition interne, disciplinairement autonome, des savoirs. Et cette requalification, en apparence autonome, s'effectue en connexion avec des dynamiques qui relèvent de l'environnement extérieur aux disciplines, facteur de leur ébranlement. Les requalifications intradisciplinaires ont donc à faire avec des déterminations hétéronomes et hors disciplines, désormais incalculables: elles n'ont pas été décidées ou pensées de manière souveraine par des maîtres à penser. C'est plutôt le postulat d'une telle autorité du savoir, ou des sujets de savoir, qui a pris fin, la péremption étant lisible dans la « pertinence épistémologique " ${ }^{36}$, à des fins de requalification, d'une notion telle que gender. Ces dynamiques ont à voir avec la dynamique d'un tout-monde qui largue les amarres, s'éloigne d'un périmètre disciplinaire, d'un empire des savoirs qu'on connaissait naguère plus restreint, s'agissant du bornage chronologique et des extensions spatiales. A ces dynamiques d'ébranlement hors champ-changement climatique, crise économique et sociale à l'échelle d'un monde, désormais explicitement et spectaculairement interconnecté- nos disciplines ne peuvent pas ne pas réagir, en un processus de requalification qui ne fait que s'ouvrir, la reconfiguration offrant une réplique intraacadémique qui tente d'encaisser et de penser l'ébranlement du dehors. Cela se traduit par l'invocation d'une histoire connectée, qui doit envisager des étendues et des durées temporelles bien plus amples, ou par l'ouverture aux interrogations de la post-colonialité, avec toutes les relectures et requalifications qu'impose un tel horizon des possibles ${ }^{37}$.

\section{Conclusion : Illusio salvatrice?}

Publié à la suite de La Distinction (1979), homo academicus (1984), avec sa cartographie des champs dominants et dominés qui structurent l'habitat académique, puis des champs emboîtés qui, à leur tour, comme des poupées russes, imposent une domination relative au sein d'un champ lui-même dominé, est un livre ayant des propriétés de désenchantement comique ${ }^{38}$. Méditations pascaliennes est un livre qui pose une question plus vive, plus cruelle. Car si on peut mesurer le gain qu'octroie homo academicus à l'agent académique, ainsi pourvu d'une plus grande lucidité, il reste la question de ce qui fait marcher ou fait tenir l'agent d'un savoir, dans la pratique d'un métier académique, dans la poursuite des opérations permettant la réalisation de sa libido sciendi. La question est d'une acuité particulière, dès lors que la pratique d'enseignement et de recherche se fait au sein d'une université publique et dans le domaine disciplinaire des sciences sociales :

31 L'implicite en ce cas, c'est ce qui est impliqué dans le fait d'être pris au jeu, c'est-à-dire dans l'illusio comme croyance fondamentale dans l'intérêt du jeu et la valeur des enjeux qui est inhérente à cette appartenance. L'entrée dans un univers scolastique suppose une mise en suspens des présupposés du sens commun et une adhésion para-doxale à un ensemble plus ou moins radicalement nouveau de présupposés, et, corrélativement, la découverte d'enjeux et d'urgences inconnus et incompris de l'expérience ordinaire. Chaque champ se caractérise en effet par la poursuite d'une fin spécifique, propre à favoriser des investissements tout aussi absolus chez tous ceux (et ceux-là seulement) qui 
possèdent les dispositions requises (par exemple, la libido sciendi). Participer de l'illusio, scientifique, littéraire, philosophique ou autre, c'est prendre au sérieux (parfois au point d'en faire, là aussi des questions de vie et de mort) des enjeux qui, nés de la logique du jeu lui-même, en fondent le sérieux, même s'ils peuvent échapper ou paraître " désintéressés » et " gratuits » à ceux que l'on appelle parfois les " profanes »"

Le «jeu» de production de ces savoirs spécifiques, par définition hors de portée ou de compréhension du "profane ", demande un investissement d'affect (libido sciendi), dont l'intensité est corrélée à sa probable inopérabilité en dehors du champ : l'agent du champ est doté d'un charme dont l'efficace intra-champ s'arrête à la frontière disciplinaire. Ayant acquitté un droit d'entrée, celui-ci est payé en retour d'un gage symbolique dont le cours est par définition local, interne au champ.

Or s'agissant d'un champ qui est défini par l'étendue d'une aire, caractérisé par l'élévation des droits d'entrée et, de manière concomitante, sa mise en conformité avec les clés d'entrée exigibles dans d'autres champs disciplinaires des sciences sociales, on peut identifier au moins quatre propriétés possibles, constitutives d'une illusio qui peut faire tenir : 1) l'adhésion à une déontologie générale de service public, où l'impératif de spécialisation, toujours plus pointue, des savoirs accumulés par l'agent s'équilibre d'un postulat quant à la transmission des savoirs et quant à la valeur civique et émancipatrice de leur détention par les usagers que sont les étudiants; 2) le plaisir pris au jeu, à la pureté ou à la beauté de l'émulation rivalitaire qui structure un champ où « l'accroissement de l'homogénéité entre les concurrents $»^{40}$ a pour corrélat l'intensification des microdistinctions; 3) le postulat d'utilité sociale immédiate, s'agissant de l'apport au débat médiatique d'une discipline portant sur une aire géopolitiquement majeure pour la compréhension du monde contemporain; 4) la participation à la construction d'un savoir critique et transmissible, préalable à une transformation sociale générale, dont la nécessité est à la fois postulée et catalysée par la critique que construit l'agent.

Aucun agent ne pourra invoquer simultanément l'ensemble de ces propriétés. La $4^{\mathrm{e}}$ s'entend comme une radicalisation urgente et conjoncturelle de la $1^{\text {ère }}$. La $2^{\mathrm{e}}$ relève de l'habitus qui a été consolidé à travers la reconstruction du champ de la civilisation britannique au cours des dernières décennies, dans un environnement plus exigeant et plus concurrentiel. La $3^{\mathrm{e}}$ (offrant la surface sociale et profane d'une illusio médiatique) peut compromettre l'autorité disciplinaire requise pour le plein exercice de l'autorité intra-champ, à travers le plaisir pris au jeu savant et exigeant, inaccessible au profane.

La fin de partie, la sortie du jeu distinctif propre à l'habitat académique, s'est toujours reconnue, avant et depuis la péremption de son espace de "liberté subjective», au dépérissement terminal de l'illusio, motrice ou salvatrice. Des propos symptomatiques de l'imminence d'une telle levée d'illusio sont parfois audibles à la fin d'un comité de sélection, après évaluation des candidatures ayant satisfait à des droits d'entrée profondément modifiés et rehaussés, en peu de temps, selon une dynamique qui est à l'image de la mutation du monde : si j'avais été candidat.e, je n'aurais pas été recruté.e. Cela peut se dire avec une pointe de schadenfreude. Dans ce cas, l'aveu reste pris dans l'illusio, dont le charme opère tant qu'un reste de complaisance narcissique tient encore. Or la règle implacable, systémique, est celle d'un dépérissement final de toute illusio, sans compensation possible sur une autre scène: cela est la loi des champs soumis à une dynamique de reconstruction et de reconfiguration, que l'on a pu appréhender à travers l'exercice du métier universitaire, comme si l'effet salutaire d'un parcours professionnel 
est à la fin l'extinction de l'illusio qui a été le concomitant de l'habitus provisoirement maintenu au cours d'une carrière.

Il me vient cependant, à la lecture d'un propos lapidaire d'Emmanuel Levinas, cité par Denis Guénoun, "L'expérience, l'idée de l'infini, se tient dans le rapport avec Autrui. L'idée de l'infini est le rapport social $»^{41}$, une illusio finale. Celle de vouloir envisager la possibilité d'une modalité d'échange, d'enseignement et d'interrogation, où «le rapport social » ne serait pas invoqué seulement comme un déterminant de destin, comme une catégorie molaire et impensée, propice à l'assignation rigide et identitaire.

Peut-on envisager sous "l'idée de l'infini " l'étude «du rapport social», ici celui qui est spécifique au terrain de la civilisation britannique ? D'un terrain par définition ouvert sur le tout-monde, à travers une exploration conduite depuis ce point d'extériorité proche qu'est le lieu francophone? Cela est peut-être une illusio sans illusion : plaidoyer pour une interrogation sur le monde sous forme d'une pratique disciplinaire que l'on souhaiterait garder ouverte, à destination des étudiants en cours et des nouvelles générations lancés dans la recherche en civilisation britannique, donc en " rapport social » : avec "l'idée de l'infini $»^{42}$ comme obligation épistémologique, s'agissant de l'objet et de la méthode.

Cornelius Crowley (CREA EA 370) est professeur de civilisation britannique à l'université Paris Nanterre. Publications récentes : «Foucault traducteur, Foucault traduit ", dans Claire Wrobel (dir.), Traduction et philosophie, Editions PanthéonAssas, 2018 ; " A British Grammar of Belief and Self-Belief", revue Babel, $n^{\circ}$ XIII, Croyances contemporaines : regards croisés sur le monde anglophone (dir. Frédéric Armao), Université de Toulon, 2017.

\section{BIBLIOGRAPHIE}

BERNAS Colette, GAUDIN Elizabeth, POIRIER François, Le document de civilisation britannique, lecture analyse, commentaire, Grenoble, Ophrys, 1992.

BOLTANSKI Luc, Énigmes et complots, une enquête à propos d'enquêtes, Paris, Gallimard, 2012.

BOURDIEU Pierre, homo academicus (1984), Paris, Éditions de Minuit, 2002.

BOURDIEU Pierre , Méditations pascaliennes (1997), Paris, Éditions du Seuil, 2003.

BOUVERESSE Jacques, Nietzsche contre Foucault, Sur la vérité, la connaissance et le pouvoir, Paris, Agone, 2016.

COLLINI Stefan, Speaking of Universities, London, Verso, 2017.

COLLINI Stefan, What Are Universities For?, Harmondsworth, Penguin, 2012.

COLLINI Stefan, « HiEdBiz », London Review of Book, vol. 25, n 21, 6 novembre 2003, https:// www.lrb.co.uk/v25/n21/stefan-collini/hiedbiz (site consulté le 13 juillet 2018).

DUBOIS Pierre, « Histoires d'universités », https://histoiresduniversites.wordpress.com/ (consulté le 13 juillet 2018).

FOSTER Roy, « Will the Center Hold?», The New York Review of Books, vol. LXV, n 12, juillet 2018. 
GIRARD René, La Violence et le sacré, Paris, Grasset, 1972.

GUÉNOUN Denis, Des verticales dans l'horizon: Six croisements entre philosophie et théologie, Genève, Labor et Fides, 2018.

GUY Stéphane, Bernard Shaw et la Société Fabienne. Aspects du militantisme socialiste britannique au tournant du vingtième siècle, trois volumes, Université Sorbonne Nouvelle, 2018.

HOGGART Richard, The Uses of Literacy: Aspects of Working Class Life (1957), Harmondsworth, Penguin, 2009.

KIBERD Declan, After Ireland: Writing the Nation from Beckett to the Present, Cambridge Mass., Harvard UP, 2018.

LUHMANN Niklas, Observations on Modernity, trad. Whobrey William, Standford, Stanford UP, 1998.

MATUSZEK Krzystof, « Ontology, reality and construction in Niklas Luhmann's Theory », Constructivist Foundations 10 (2), 2015, http://constructivist.info/10/2/203 (site consulté le 13 juillet 2018).

PÉREZ Joseph, Andalousie, Vérités et légendes, Paris, Tallandier, 2018.

O' DAY Alan, « Max Weber and leadership : Butt, Parnell and Dillon : nationalism in transition », in BOYCE D. George et O'DAY Alan, (dir.), Ireland in Transition, 1867-1921, London, Routledge, 2004.

PIRON Stéphane, L'occupation du monde, Paris, Éditions Zones sensibles, 2018.

POIRIER François, Génération Thatcher, la culture politique de l'Angleterre, St. Denis, Presses universitaires de Vincennes, 1992.

RIOT- SARCEY Michèle, «Le genre en histoire : une question de méthode », in BRAILOWSKY Yan et INGLEBERT Hervé (dir.), 1970-2010 : les sciences de l'Homme en débat, Nanterre, Presses universitaires de Nanterre.

http://www.agence-nationale-recherche.fr/, (consulté le 13 juillet 2018).

Combattre pour le Socialisme n68 (juin 1997), http://socialisme.free.fr/bulletin/ cps68_universite.htm (consulté le 13 juillet 2018).

http://congres2018.saesfrance.org/panels-interdisciplinaires/ (consulté le 13 juillet 2018).

https://www.diplomatie.gouv.fr/IMG/pdf/enseignement_superieur.pdf (consulté le 13 juillet 2018).

https://ec.europa.eu/education/policy/higher-education/bologna-process_fr (consulté le 13 juillet 2018).

https://evaluation.hypotheses.org/63; https://www.cne-evaluation.fr/index.html, (consulté le 13 juillet 2018).

https://www.hceres.fr/, (consulté le 13 juillet 2018).

http://www.iufrance.fr/les-statuts-de-liuf.html, (consulté le 13 juillet 2018).

https://www.lesechos.fr/31/01/1992/LesEchos/16068-062-ECH_bouclage-du-planuniversites-2000.htm (consulté le 13 juillet 2018). 


\section{NOTES}

1. La notion de construction est d'une importance cruciale, s'agissant des enjeux de méthode et d'objet dans le champ général des sciences sociales, donc en civilisation britannique. Voir MATUSZEK Krzystof, "Ontology, reality and construction in Niklas Luhmann's Theory ", Constructivist Foundations 10 (2), 2015, p. 203-210. http://constructivist.info/10/2/203 (site consulté le 13 juillet 2018). Pour la critique d'un postulat de "constructivisme radical», voir BOUVERESSE Jacques, Nietzsche contre Foucault, Sur la vérité, la connaissance et le pouvoir, Paris, Agone, 2016, p. 125.

2. "If we describe contemporary society structurally according to sociological tradition as a functionally differentiated system, then it follows that the differentiated functions systems, having become autonomous, distinguish themselves from their surroundings (both intrasocial and extrasocial) ", LUHMANN Niklas, Observations on Modernity, trad. WHOBREY William, Standford, Stanford UP, 1998, p. 9-10. La différenciation est, pour Luhmann, le trait distinctif de la modernité ou d'une modernisation. Un tel processus de différenciation s'observe dans la reconstruction de la civilisation britannique.

3. La catégorie d'environnement, par rapport auquel un système se démarque, est cruciale dans la théorie des systèmes de Luhmann, Ibid., p. 108-113.

4. BOURDIEU Pierre, Méditations pascaliennes (1997), Paris, Éditions du Seuil, 2003, p. 237-295.

5. Ibid., p. 213.

6. COLLINI Stefan, Speaking of Universities, London, Verso, 2017; What Are Universities For?, Harmondsworth, Penguin, 2012, et une série d'articles publiés sur la question dans London Review of Books, depuis 2003 : « HiEdBiz », LRB, vol. 25, n 21, 6 novembre 2003 : https://www.lrb.co.uk/ $\mathrm{v} 25 / \mathrm{n} 21 /$ stefan-collini/hiedbiz (site consulté le 13 juillet 2018).

7. A propos des luttes au sein d'un champ scientifique, Bourdieu écrit : "L'objectivation de ces luttes, et le modèle de la correspondance entre l'espace des positions et l'espace des prises de position qui en dévoile la logique, sont le produit d'un travail armé d'instruments de totalisation et d'analyse (comme la statistique) et orienté vers l'objectivité, horizon ultime, mais sans cesse reculé, d'un ensemble de pratiques collectives que l'on peut décrire avec BACHELARD Gaston, comme un effort constant de désubjectivation ». BOURDIEU Pierre, Méditations pascaliennes (1997), Paris, Éditions du Seuil, 2003, p. 171.

8. BOURDIEU Pierre, homo academicus (1984), Paris, Éditions de Minuit, 2002.

9. Le football est assurément un jeu de terrain ou de champ qui a progressivement développé un système de jeu. A cet égard, le récit mythique ou mémoriel du football tend à reproduire le schéma pointé par Bourdieu : la nostalgie d'une « liberté concrète » incarnée. Un tel schéma, wéberien, est convoqué dans un article d'O'Day Alan, «Max Weber and leadership: Butt, Parnell and Dillon: nationalism in transition ", consacré à l'évolution du parti parlementaire irlandais à Westminster dans la période 1870-1914, BOYCE D. George, O'DAY A. (dir.), Ireland in Transition, 1867-1921, London, Routledge, 2004, p. 17-34.

10. Dans la consolidation du champ de la civilisation américaine, celle-ci a pu bénéficier de l'intérêt pour les lieux et moments de la contestation en Amérique. S'agissant de la civilisation britannique, l'avènement de Margaret Thatcher, personnalité clivante, a sans doute accru l'intérêt pour les études en civilisation britannique. Citons, à titre de jalons, le livre de POIRIER François, Génération Thatcher, la culture politique de l'Angleterre, St. Denis, Presses universitaires de Vincennes, 1992, ainsi que le volume publié par BERNAS Colette, GAUDIN Elizabeth, POIRIER François, Le document de civilisation britannique, lecture analyse, commentaire, Grenoble, Ophrys, 1992.

11. BOURDIEU Pierre, Méditations pascaliennes (1997), Paris, Éditions du Seuil, 2003, p. 213.

12. Dans le document de synthèse de son dossier d'HDR, dont le titre d'ensemble est « Bernard Shaw et la Société Fabienne. Aspects du militantisme socialiste britannique au tournant du vingtième 
siècle ", Université Sorbonne Nouvelle, 2018, Stéphane Guy commente l'approche développée par Stephen Greenblatt en 1980, dans son livre Renaissance Self-Fashioning. "Parce qu'une culture se caractérise par des 'interactions de sens', la littérature constitue, pour Greenblatt, le lieu d'une expérience du réel au même titre que d'autres discours...de sorte que la tâche du chercheur consisterait à se défaire des frontières disciplinaires pour tenter de restituer la complexité de la culture où les œuvres, quelles qu'elles soient, sont produites » (p. 47).

13. "Aboutissement de la réflexion critique sur la pratique scientifique...l'analyse sociologique du monde universitaire vise à faire tomber l'Homo Academicus, classificateur entre les classificateurs, dans ses propres classements ». BOURDIEU Pierre, "Postface : vingt ans après ", homo academicus, Paris, Éditions de Minuit, 2002, p. 289.

14. https://www.lesechos.fr/31/01/1992/LesEchos/16068-062-ECH_bouclage-du-planuniversites-2000.htm (consulté le 13 juillet 2018).

15. Le blog de DUBOIS Pierre, "Histoires d'universités", constitue depuis dix ans un lieu d'observation des débats dans les universités concernant les transformations institutionnelles, https://histoiresduniversites.wordpress.com/ (consulté le 13 juillet 2018).

16. Le gouvernement dirigé par Alain Juppé, dans lequel François Bayrou était ministre de l'Éducation nationale, a démissionné après la défaite de la droite lors des élections législatives du 1 juin 1997. Pour une critique sans concession de la réforme, alors que la question posée était de savoir si le successeur de Bayrou allait poursuivre avec la semestrialisation, voir le texte publié dans Combattre pour le Socialisme $n^{\circ} 68$ (juin 1997), http://socialisme.free.fr/bulletin/ cps68_universite.htm (consulté le 13 juillet 2018).

17. BOURDIEU Pierre, Méditations pascaliennes (1997), Paris, Éditions du Seuil, 2003, p. 170.

18. Ibid., p. 170.

19. «Un événement énigmatique peut bien avoir, par là, une signification immédiate (tel immeuble s'est effondré), au sens où le changement d'état... peut faire l'objet d'une description faisant appel à des données physiques généralement admises [...]. Mais on peut dire qu'il ne possède pas de sens tant qu'il n'a pas été possible de l'attribuer à une certaine entité ou, lorsque cette entité est déjà connue, d'en déterminer les intentions». BOLTANSKI Luc, Énigmes et complots, une enquête à propos d'enquêtes, Paris, Gallimard, 2012, p. 23.

20. https://ec.europa.eu/education/policy/higher-education/bologna-process_fr (consulté le 13 juillet 2018).

21. https://www.diplomatie.gouv.fr/IMG/pdf/enseignement_superieur.pdf (consulté le 13 juillet 2018).

22. https://evaluation.hypotheses.org/63; https://www.cne-evaluation.fr/index.html, (consulté le 13 juillet 2018).

23. https://www.hceres.fr/, (consulté le 13 juillet 2018).

24. http://www.iufrance.fr/les-statuts-de-liuf.html, (consulté le 13 juillet 2018).

25. http://www.agence-nationale-recherche.fr/, (consulté le 13 juillet 2018).

26. https://erc.europa.eu/, (consulté le 13 juillet 2018).

27. Lors de la table ronde intitulée «Les études anglaises au contact des sciences sociales: De l'hégémonie occidentale à la montée en puissance d'autres épistèmes", Congrès 2018 de la SAES, Nanterre, 7-9 juin, François Cusset a évoqué un rôle de "sas", d'espace ou champ de relation, pour les études anglophones au sein de l'université française, http://congres2018.saesfrance.org/ panels-interdisciplinaires/ (consulté le 13 juillet 2018).

28. BOURDIEU Pierre, Méditations pascaliennes (1997), Paris, Éditions du Seuil, 2003, p. 213.

29. Je citerai le propos suivant, qui s'appuie sur le propos d'un helléniste, Fernand Robert. Si la clarté binaire me parait contestable, il s'agit simplement de constater la redoutable complexité de tout travail de compréhension d'une aire identifiée en référence à la pluralité linguistique. " Qu'est-ce qui distingue les langues vivantes des langues mortes? On étudie les premières pour connaître ce qui est différent de nous. Au contraire, 'quand il s'agit de l'Antiquité, notre attention va directement à peu 
près exclusivement aux ressemblances [...]' ». PÉREZ Joseph, Andalousie, Vérités et légendes, Paris, Tallandier, 2018, p. 111.

30. Voir la recension par l'historien FOSTER Roy, dans The New York Review of Books, juillet 2018, du livre de KIBERD Declan, After Ireland, Writing the Nation from Beckett to the Present, Cambridge (Mass.), Harvard UP, 2018. Pour Kiberd, une exceptionnalité littéraire semble supposer une exceptionnalité de la culture nationale. Ainsi une normalisation de l'Irlande s'entendrait comme extinction d'une culture d'exception. Le schéma fondamental adopté par Kiberd n'est pas éloigné de celui qui motive la déploration audible dans le livre de HOGGART Richard, The Uses of Literacy (1957) à propos d'une authenticité culturelle, en péril, de la classe ouvrière anglaise.

31. L'hypothèse rivalitaire relève du paradigme construit par René Girard, notamment à partir de La Violence et le sacré, Paris, Grasset, 1972. La violence mimétique ne prend pas fin avec l'entrée dans la différenciation qui est le propre de la modernité. Bien au contraire : si on associe Weber, Luhmann et Freud à Girard, cela relève de l'évidence.

32. « Une étude universitaire sur le militantisme politique se heurte nécessairement à la question de la discipline scientifique dans laquelle le chercheur entend se situer. A la différence d'une recherche sur la théorie qui s'inscrirait dans le champ de la philosophie politique, ce concept suppose une pensée en action et donc la conjugaison d'idées avec leur mise en œuvre. L'étude simultanée d'un processus intellectuel, d'une construction théorique, d'une réflexion sur des catégories telles que le pouvoir, la société, la propriété et le peuple, et, d'autre part, de la pratique selon des modalités spécifiques, dans un contexte déterminé par l'économie, le débat politique, les relations entre les individus, la culture ou encore les représentations collectives. » GUY Stéphane, Ibid., p. 3.

33. GUÉNOUN Denis, Des verticales dans l'horizon: Six croisements entre philosophie et théologie, Genève, Labor et Fides, 2018, p. 29.

34. BRAILOWSKY Yan et INGLEBERT Hervé (dir.), 1970-2010: les sciences de l'Homme en débat, Nanterre, Presses universitaires de Nanterre, 2013.

35. RIOT-SARCEY Michèle, «L genre en histoire : une question de méthode », Ibid., p. 177-188.

36. Ibid., p. 177.

37. PIRON Stéphane, L'occupation du monde, Paris, Éditions Zones sensibles, 2018.

38. "Situation de comédie, celle du trompeur trompé, de l'arroseur arrosé, que certains, pour se faire peur ou pour faire peur, aiment à prendre au tragique ». BOURDIEU Pierre, homo academicus (1984), Paris, Éditions de Minuit, 2002, p. 289.

39. BOURDIEU Pierre, Méditations pascaliennes (1997), Paris, Éditions du Seuil, 2003, p. 25.

40. Ibid., p. 170.

41. GUÉNOUN Denis, Des verticales dans l'horizon: Six croisements entre philosophie et théologie, Genève, Labor et Fides, 2018, p. 46.

42. Ibid., p. 46.

\section{RÉSUMÉS}

L'article examine l'évolution de la discipline de la civilisation britannique sur la période 1990-2018. L'hypothèse avancée est celle d'une reconstruction, se traduisant par un plus grand rayonnement, corrélée à une scientificité plus exigeante, conditionnée par une "élévation progressive des droits d'entrée» (Bourdieu 2003) dans le champ. L'article s'interroge sur les 
possibles évolutions à venir, dans un champ académique en profonde mutation, du fait notamment des ébranlements en cours dans l'ordre du monde.

The article examines evolutions in British civilisation studies in the period 1990-2018. The hypothesis advanced is that of a reconstruction of the discipline, evident in its enhanced visibility, coupled with the application of more demanding criteria of scientific demonstration and a "gradual elevation of the "entrance rights" (Bourdieu 2003) required for admission to the field. The article reflects on the possible future evolutions, in an academic field subject to profound mutational factors, in particular due to the tremors currently registered in the order of the world.

INDEX

Keywords : British civilisation, construction, Bourdieu, area studies

Mots-clés : civilisation britannique, construction, Bourdieu, aire, discipline

\section{AUTEUR}

\section{CORNELIUS CROWLEY}

CREA EA 370, Université Paris Nanterre 